\title{
Preface of the Special Issue of the Sixth French-Speaking Conference on Ontologies
}

\section{Gayo Diallo ${ }^{1}$. Fleur Mougin ${ }^{1}$}

Published online: 29 October 2018

c) Springer-Verlag GmbH Germany, part of Springer Nature 2018

The key role played by ontologies to solve interoperability issues is nowadays well established. They convey the necessary semantics which helps understanding, sharing and integrating heterogeneous information. The current issue of the Journal on Data Semantics publishes extended versions of selected papers from the 6th edition of the FrenchSpeaking Conference on Ontologies (JFO) held from 13 to 14th October 2016 in Bordeaux, France. All the extended papers went through an additional peer review process. As a result, three out of five selected papers have been accepted for publication in this issue.

The first paper Harnessing the power of unified metadata in an ontology repository: the case of AgroPortal, by Clement Jonquet, Anne Toulet, Biswanath Dutta and Vincent Emonet, addresses the issue of describing knowledge resources included in ontology repositories and portals using metadata. The study reviews 23 existing standards and vocabularies to describe metadata for ontologies and suggests a simplified model of 127 properties for the description of ontologies. They have been implemented in the context of the ontology repository AgroPortal that is based on the NCBO technology.
The second paper, entitled Imaging Biomarker Ontology (IBO): a biomedical ontology to annotate and share imaging biomarker data and authored by Emna Amdouni and Bernard Gibaud, deals with the design of a model for radiological measurements. They propose a semantic representation of imaging biomarkers articulated through the following three axes: the measured quality, the measurement tool and the decision tool. As an illustration, the resulting ontology has been used to annotate quantitative and qualitative imaging data from The Cancer Genome Atlas.

The third paper Dealing with direct and indirect ontology alignment, by Marouen Kachroudi and Sadok Ben Yahia, proposes two approaches for, respectively, dealing with direct cross-lingual ontology alignment and indirect alignment. While they brought techniques inspired by the information retrieval domain for cross-lingual alignment identification, an alignment composition system is introduced in order to produce indirect alignments.

Acknowledgements We would like to thank all the reviewers who read carefully the different papers and helped to improve significantly their quality.
Gayo Diallo

gayo.diallo@u-bordeaux.fr

Fleur Mougin

fleur.mougin@u-bordeaux.fr

1 Team ERIAS, BPH Centre INSERM 1212, University of Bordeaux, 33000 Bordeaux, France 